\title{
Pneumoperitônio idiopático em idoso - Relato de Caso
}

\section{Idiopathic Pneumoperitoneum in the Elderly - Case Report}

Renato Nogueira do Amaral ${ }^{1}$, Luciana Cavalcanti Valadares ${ }^{2}$, Renato Vilasboas Antunes ${ }^{2}$, Bárbara Pessoa de Matos ${ }^{2}$, Andréia Farias Alquimim², Gustavo Mazon ${ }^{2}$

1. Radiologista chefe no Hospital das Clínicas Samuel Libânio, Pouso Alegre (Minas Gerais), Brasil. 2. Residentes em radiologia no Hospital das Clínicas Samuel Libânio, Pouso Alegre (Minas Gerais), Brasil.

\section{Resumo}

Introdução: 0 pneumoperitônio frequentemente é causado por perfurações de vísceras ocas, mas, quando não se identifica uma etiologia, é dito idiopático Relato de caso: Paciente com 80 anos, admitido com quadro de dor abdominal, em pontada, contínua, especialmente em quadrante inferior esquerdo e parada de eliminação de gases e fezes há seis dias. Apresentava-se sudorético, taquicárdico, normotenso, ausculta cardiopulmonar inocente. Passado de colecistectomia e apendicectomia. Exames laboratoriais inalterados. Radiografia e tomografia computadorizada de abdome evidenciaram pneumoperitônio em região subfrênica direita. Paciente foi submetido à laparotomia exploradora e não foram evidenciados sinais de perfuração de víscera oca pós-busca minuciosa da etiologia. Conclusão: Trata-se realmente de causa idiopática, tendo evoluído bem após o procedimento. Entretanto, não há consenso em relação ao melhor tipo de intervenção.

Palavras-chave: Pneumoperitônio. Idiopático. Idoso

\begin{abstract}
Introduction: Pneumoperitoneum is often caused by perforations of hollow viscera, but when an etiology is not identified, it is said to be idiopathic. Case report: A patient with 80 years of age, admitted with continuous abdominal pain, especially in the lower left quadrant and Stopping gas and stool for six days. She presented sweating, tachycardia, normotensive, and innocent cardiopulmonary auscultation. Past Cholecystectomy and appendectomy. Unchanged laboratory tests. Radiography and computed tomography of the abdomen showed pneumoperitoneum In the right subphrenic region. Patient was submitted to exploratory laparotomy and no signs of hollow viscera perforation were found after search The etiology. Conclusion: This is indeed an idiopathic cause, having evolved well after the procedure. However, there is no consensus Relation to the best type of intervention.
\end{abstract}

Key words: Pneumoperitoneum. Idiopathic. Elderly.

\section{INTRODUÇÃO}

O pneumoperitônio frequentemente é causado por perfurações de vísceras ocas, mas, quando não se identifica uma etiologia, é dito idiopático1, podendo estar presente em aproximadamente 10\% dos pneumoperitônios ${ }^{1}$.

Ele pode ser originário de processos intratorácicos, abdominais, ginecológicos e até iatrogênicos ${ }^{2}$; no entanto, não foi observada relação do estudo relatado com outras desordens agudas associadas. Desse modo, pretendemos relatar um caso de pneumoperitônio idiopático em um paciente de 80 anos, devido ao fato de ser um achado incomum no dia a dia da prática médica, como também na literatura, além de oferecer um suporte teórico-prático na investigação etiológica de casos de pneumoperitônio na prática médica.

\section{RELATO DE CASO}

O Paciente B. L. O, 80 anos, admitido com quadro de dor abdominal, em pontada, contínua, especialmente em quadrante inferior esquerdo e parada de eliminação de gases e fezes há seis dias. Apresentava-se sudorético, taquicárdico, normotenso, ausculta cardiopulmonar inocente.

Passado de colecistectomia e apendicectomia. Negava história pregressa de outras patologias, bem como ingesta alcoólica e tabagismo. Exames laboratoriais inalterados (hemograma, enzimas hepáticas e pancreáticas, urina rotina, ureia, creatinina, LDH). Radiografia (figura A) e tomografia computadorizada (figura $B$ e C) de abdome evidenciaram pneumoperitônio em região subfrênica direita. Paciente foi submetido à laparotomia exploradora devido ao risco de complicações na demora em abordar, o que tornaria o manejo do paciente mais difícil. Não foram evidenciados sinais de perfuração de víscera oca pós-busca minuciosa da etiologia, concluindo-se, assim, que a causa é realmente idiopática. $O$ paciente evoluiu bem após o procedimento, com alta hospitalar após triagem laboratorial e de imagem inocentes. $O$ paciente foi informado da divulgação de seu caso, dando seu consentimento. 
Figura 1. Radiografia simples (A) e Tomografia computadorizada (B e C) do abdome evidenciando pneumoperitônio em região subfrênica direita $424 \times 318 \mathrm{~mm}(72 \times 72 \mathrm{DPI})$

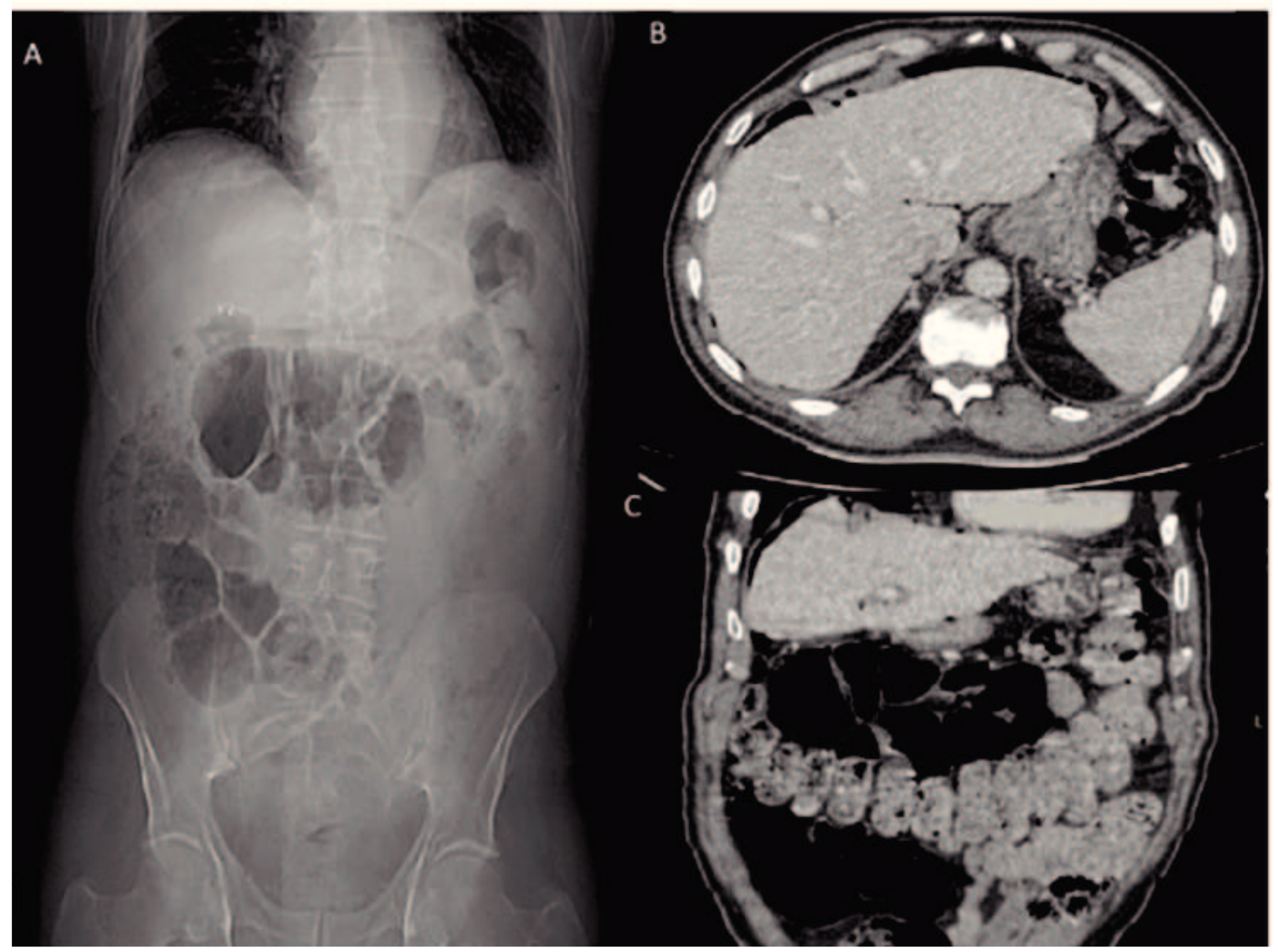

\section{DISCUSSÃO}

O pneumoperitônio, em geral, origina-se de causas cirúrgicas e não cirúrgicas (principalmente abdominal, torácica e ginecológica), na maioria das vezes devido à úlcera gástrica ou duodenal perfurada, mas em $10 \%$ dos casos pode ser de etiologia idiopática, caracterizando-se por condição rara, havendo uma média de vinte relatos na literatura ${ }^{1,2}$. O pneumoperitôneo pósoperatório pode persistir por até quatro semanas, embora a reabsorção completa ocorra dentro da primeira semana.

A faixa etária observada para tais pacientes é bastante variável, sendo a maioria vista desde a infância até a oitava década de vida, como no caso relatado.

A causa mais frequente tende a ser de origem abdominal, destacando-se pneumatose cística intestinal, abscesso hepático, microtraumas de transição esofagogástrica por vômitos, colite e trauma da parede gástrica após hiperinsuflação em manobras de ressuscitação cardiopulmonar. Quanto às causas abdominais não cirúrgicas, pode-se citar história de procedimentos endoscópicos, punções percutâneas ou enemas ${ }^{3}$.

Entre as causas intratorácicas podem ser incluídas: trauma, barotrauma, adenotonsilectomia, tuberculose pulmonar, fístula broncopulmonar, ruptura espontânea de bolhas pulmonares, pneumotórax, podendo estar associada a pneumomediastino ou pneumocárdio, havendo ainda associação com a ventilação mecânica em pacientes com terapia intensiva após ressuscitação cardiopulmonar e após aumento da pressão intratorácica, por exemplo, tosse, vômito ou manobra de Valsalva $a^{4,5,6}$.

O diagnóstico radiológico pode ser feito inicialmente por meio de radiografia de abdome, a qual evidencia o pneumoperitônio quando o volume de ar na cavidade está acima de um litro4, sendo considerável quando detectado em exames, como no caso do paciente em questão. Mas, também, em grande parte dos casos, o diagnóstico é realizado por tomografia computadorizada, já que muitos desses pacientes apresentamse sintomáticos, tendo sido realizados protocolos para abdome agudo.

Após a confirmação radiológica, geralmente pode-se optar por uma conduta cirúrgica (laparotomia exploradora), como foi feito com o paciente em questão, sendo também conduta a adotada em todos os casos referenciados, para a pesquisa 
do foco da possível perfuração. No entanto, essa conduta é preferencialmente observada nos casos relacionados a sintomas de peritonite associada.

Vale ressaltar que, na revisão da bibliografia, evidenciou-se uma dualidade de aspectos clínicos apresentados pelos pacientes, uns com quadro inflamatório associado sem foco perfurativo, e outros em que o único achado foi o pneumoperitôneo.

Embora a apresentação do pneumoperitôneo seja frequentemente sugestiva de patologia cirúrgica significativa, existem casos em que pode ser adotada conduta conservadora e assim, evitar os riscos de se submeter a uma laparotomia5,7. Apesar da apresentação clínica do paciente em questão e dos achados radiográficos, na ausência de instabilidade cardiovascular, é possível monitorar esses pacientes, tentando abordagem conservadora, a qual não foi adotada para a prevenção de possíveis complicações ${ }^{5}$.

\section{CONCLUSÃO}

O pneumoperitôneo idiopático é uma condição rara tanto no dia a dia, quanto na literatura e, mesmo em pacientes sintomáticos, sem exames laboratoriais alterados, geralmente é prescrita uma conduta cirúrgica a fim de fazer uma melhor avaliação de um possível foco perfurativo. Tal evolução ainda é geradora de opiniões divididas na literatura, com o objetivo de tentar inicialmente uma conduta conservadora com tais pacientes, a fim de evitar complicações advindas da cirurgia.

\section{REFERÊNCIAS}

1. Amico, EC, Vivas DV, Alves JR. Pneumoepritônio idiopático: relato de caso. Rev. Col. Bras. Cir. 2011 Mar-Abr; 38(2): 142-144. doi: http://dx.doi.org/10.1590/ S0100-69912011000200014.

2. Mularski RA, Sippel JM, Osborne ML. Pneumoperitoneum: a review of nonsurgical causes. Crit Care Med. 2000 Jul; 28(7):2638-44. PubMed PMID: 10921609.

3. Korbicka J, Capov I, Vlcek P. Pneumoperitoneum without perforation of the digestive tract. Rohzl Chir. 2002 Jul; 81(7): 364-71. PMID: 12197173.

4. Levine MS, Scheiner JD, Rubesin, SE, laufer I, Herlinger H. Diagnosis of pneumoperitoneum on supine abdominal radiographs. AJR Am J Roentgenol. 1991 Apr; 156(4): 731-5. doi: 10.2214/ajr.156.4.2003436. PubMed PMID: 2003436.
5. McLaren O. Spontaneous idiopathic recurrent pneumoperitoneum. J Surg Case Rep. 2013 Aug 29; 203(8). doi: 10.1093/jscr/rjt071. PubMed PMID: 24964474; PubMed Central PMCID: PMC3813507.

6. Tiwary S, Agarwal A, Kumar S, Khanna R, Khanna A. Idiopathic massive pneumoperitoneum. The Internet Journal of Surgery [Internet]. 2005 [dezembro 2016/ 12/08]; 8(2). Disponível em: https://ispub.com/IJS/8/2/5225.

7. Freitas WR Jr, Malheiros CA, Kassab P, Ilias EJ. Pneumoperitôneo espontâneo idiopático. Rev. Assoc. Med. Bras [Internet]. 2011 Nov-Dez [ acesso ano mês dia]; 57(6): 614. Disponível em: http://www.scielo.br/scielo.php?script=sci arttext\&pid=S0104-42302011000600004. doi: http://dx.doi.org/10.1590/ S0104-42302011000600004. 